\title{
RNA-binding activity of the rat calmodulin-binding PEP-19 protein and of the long PEP-19 isoform
}

\author{
PATRIZIA SALADINO ${ }^{1}$, CARLO MARIA DI LIEGRO $^{1}$, PATRIZIA PROIA ${ }^{2}$, ANNA SALA $^{1}$, \\ GABRIELLA SCHIERA $^{3}$, ALESSANDRA LO CICERO ${ }^{3}$ and ITALIA DI LIEGRO ${ }^{3}$
}

\author{
Dipartimenti di ${ }^{1}$ Scienze e Tecnologie Molecolari e Biomolecolari (STEMBIO), ${ }^{2}$ Studi Giuridici, Economici, \\ Biomedici, Psicosociopedagogici delle Scienze Motorie e Sportive, ${ }^{3}$ Biomedicina Sperimentale \\ e Neuroscienze Cliniche, Università degli Studi di Palermo, Palermo, Italy
}

Received September 1, 2011; Accepted September 29, 2011

DOI: $10.3892 /$ ijmm.2011.819

\begin{abstract}
Synthesis of $\mathrm{H} 1^{\circ}$ histone protein, in the developing rat brain, seems to be regulated mainly at the post-transcriptional level. Since regulation of RNA metabolism depends on a series of RNA-binding proteins, we have been searching for RNA-binding proteins involved in the post-transcriptional regulation of the $\mathrm{H} 1^{\circ}$ gene. We recently reported isolation, from a cDNA expression library, of an insert encoding a novel protein, the C-terminal half of which is identical to that of PEP-19, a brain-specific protein involved in calcium metabolism. The novel protein was called long PEP-19 isoform (LPI). Herein we show that LPI, as well as PEP-19, can bind $\mathrm{H} 1^{\circ}$ RNA. Moreover, in order to improve production of functional LPI/PEP-19, we modified the protocol normally adopted for preparing histidine tagged-proteins from bacteria, by adding an additional purification step. We also found that both LPI and PEP can compete for $\mathrm{H}^{\circ}$ RNA binding with PIPPin (CSD-C2), another RNA-binding protein previously discovered in our laboratory. Since PEP19/LPI contain a calmodulin binding domain, we finally investigated whether their ability to bind RNA is affected by calmodulin. Our results show that calmodulin interferes with binding of $\mathrm{H} 1^{\circ}$ RNA to both PEP-19 and LPI, while it is not able to bind RNA on its own. This finding suggests that calcium/calmodulin may have a role in controlling $\mathrm{H} 1^{\circ}$ mRNA metabolism in the developing brain.
\end{abstract}

\section{Introduction}

Eukaryotic DNA is packaged in a highly organized protein complex called chromatin, the smallest unit of which is the

Correspondence to: Professor Italia Di Liegro, Dipartimento di Biomedicina Sperimentale e Neuroscienze Cliniche, Sezione di Scienze Biochimiche, Università degli Studi di Palermo, via del Vespro, I-90127 Palermo, Italy

E-mail: italia.diliegro@unipa.it

Key words: RNA-binding proteins, histone variants, $\mathrm{H}^{\circ}$, PEP-19, long PEP-19 isoform, calmodulin nucleosome. Nucleosomes are formed by about 200 base pairs of DNA wrapped around a protein core, consisting of two molecules each of the histones $\mathrm{H} 2 \mathrm{~A}, \mathrm{H} 2 \mathrm{~B}, \mathrm{H} 3$ and $\mathrm{H} 4$. A fifth histone, the linker histone $\mathrm{H} 1$, binds DNA where it enters and leaves the nucleosome. Nucleosomes also provide cells with a system able to regulate gene expression, since their positioning on different parts of the gene with different functional properties can both activate or repress transcription (1). Nucleosomes can be remodeled by different mechanisms, one of which involves incorporation of specific histone variants (also called replacement histones) (2).

In the mammalian brain, regulation of stability, localization and translation of a number of transcripts plays a key role in the establishment and maintenance of nerve cell functions (3-5). Among post-transcriptionally regulated genes, those encoding histone variants, such as $\mathrm{H}^{\circ}(6-8)$ and $\mathrm{H} 3.3(2,9,10)$ are of interest for the possible involvement of histone variants in the replication-independent chromatin remodelling induced by extracellular stimuli (2). In looking for proteins able to bind mRNAs encoding the histone variants $\mathrm{H}^{\circ}$ and $\mathrm{H} 3.3$, we identified a set of proteins that bind the one and/or the other histone mRNAs (11). We also cloned a cDNA that encodes a protein, that we called PIPPin (12-14), now also known as CSD-C2, that contains a cold shock domain and binds both mRNAs at the level of the polyadenylation signal (13).

More recently, we isolated a second novel cDNA (15), encoding a putative protein of 94 amino acids, the C-terminal half (41 amino acids) of which is identical to that of PEP-19, a brain-specific protein of 62 amino acids, involved in calcium metabolism and calmodulin binding (16-18). The novel protein, that we called long PEP-19 isoform (LPI), is rat brain-specific and is likely derived by alternative splicing from the same gene as PEP-19 (15).

In order to clarify whether LPI actually is an RNA-binding protein, as suggested by the original isolation of the corresponding cDNA from an expression library by direct binding to RNA, we produced recombinant 6 histidine-tagged LPI and PEP-19. Production in bacteria of histidine-tagged proteins is, however, hampered by the fact that more than $90 \%$ of the foreign protein is recovered as unfolded, inactive form, in inclusion bodies (19). Here we report the results obtained by using, in the binding assays, LPI/PEP-19 prepared according 
to the standard protocol, normally used to purify histidinetagged proteins, to which we added a further purification step, that allows a better yield of active protein.

\section{Materials and methods}

Animals and research ethics. Sprague-Dawley rats (Stefano Morini, San Polo d'Enza, Italy) were housed in our institutional animal care facility under direction of a licensed veterinary. Procedures involving animals were conducted according to the European Community Council Directive 2010/63/EU, reducing to a minimum the use of animals.

Preparation of recombinant LPI and PEP-19 proteins, containing $N$-terminal tags of 6 histidines. Cloning of the cDNAs encoding LPI (EMBL ID: AJ493658) and PEP-19 (EMBL ID: M24852), and production of the N-terminal 6 histidine-tagged LPI and PEP-19 proteins, have been previously described (15). Recombinant PIPPin, used as a control, was prepared as previously described (20).

Chromatographic purification of recombinant LPI/PEP-19. Recombinant PIPPin, LPI and PEP-19 were purified from the insoluble fraction of bacterial homogenate by metal-affinity chromatography on a selective nickel-nitriloacetic (Ni-NTA)conjugated agarose matrix (QIAexpress System, Qiagen), according to the manufacturer's instructions. At this aim, the bacterial pellet, which contained the insoluble inclusion bodies, was resuspended in $50 \mathrm{mM}$ Tris- $\mathrm{HCl}, \mathrm{pH} 7.2 ; 50 \mathrm{mM}$ $\mathrm{NaCl}, 8 \mathrm{M}$ urea and incubated for $5 \mathrm{~min}$ at $80^{\circ} \mathrm{C}$, in order to allow protein solubilization. Proteins were allowed to refold during chromatography, by sequentially washing the column with buffers containing decreasing amounts of urea (from 8 to $0 \mathrm{M}$ ). Finally, bound proteins were eluted with $300 \mathrm{mM}$ imidazole. Specific activity of the recombinant protein was highly improved by gel filtration of the imidazole-eluted protein through an equal volume of G-100 Sephadex in $50 \mathrm{mM}$ Tris- $\mathrm{HCl}$ (pH 7.2) (patent $\mathrm{N}^{\circ}$ PA2009A000029).

Preparation of in vitro transcripts and T1 RNase protection assay. ${ }^{32} \mathrm{P}$-radiolabeled $\mathrm{H} 1{ }^{\circ}$ RNA was prepared as previously described (11), using as a template the plasmid $\mathrm{pMH} 1^{\circ}(21)$, which contains the $\mathrm{H} 1^{\circ}$ insert (EMBL ID: X70685). About $0.5 \times 10^{6} \mathrm{cpm}\left(0.5-2.0 \times 10^{7} \mathrm{cpm} / \mathrm{pmol}\right.$ of RNA $)$ of purified $\mathrm{H} 1^{\circ}$ RNA were mixed with recombinant protein (50 $\mathrm{ng}$ ), prepared as described above. For the T1 protection assay, we used a previously described method (11) except that cross-linking of RNA to proteins was performed before incubation with T1 RNase (EC 3.1.27.3; Roche). RNA-protein complexes were analyzed by denaturing electrophoresis on sodium dodecyl sulfate (SDS)-polyacrylamide slab gel (PAGE). At the end of the run, the gel was directly exposed to X-ray film for autoradiography. The gels were also stained with Coomassie Brilliant Blue R-250 (Sigma), to confirm loading of equal amounts of proteins per lane.

Competition experiments aimed at analyzing RNA-binding specificity were performed in the presence of 2:1 or 5:1 excess of unlabeled RNA. Competition experiments aimed at analyzing interference effects of LPI or PEP-19 on PIPPin (CSD-C2) binding to $\mathrm{H} 1^{\circ} \mathrm{RNA}$ were performed in the presence of $1: 1$ or
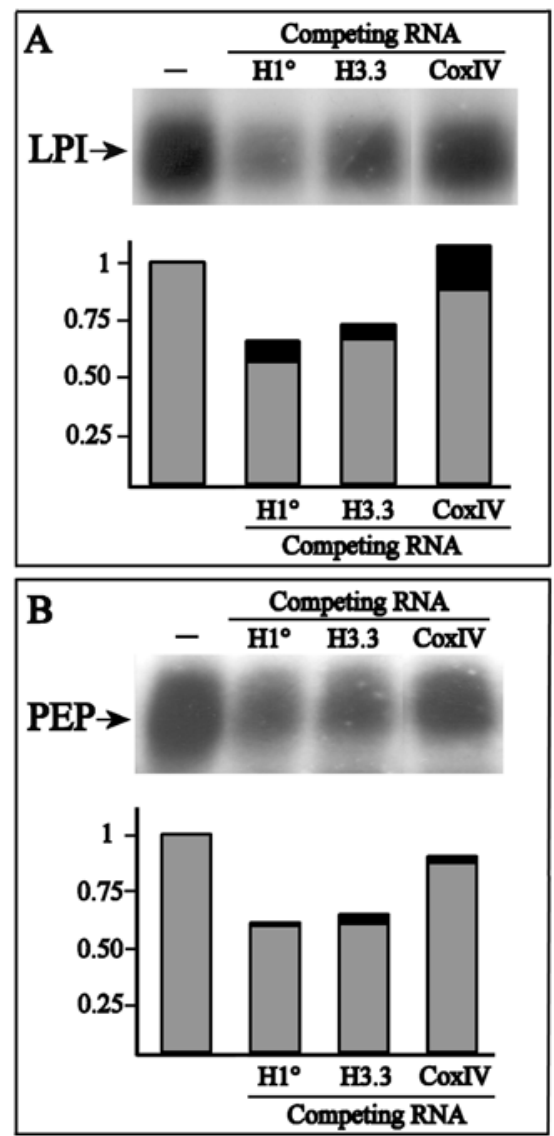

Figure 1. LPI and PEP-19 bind H1 ${ }^{\circ}$ RNA. (A) 6His-tagged LPI or (B) PEP-19 (50 ng each) were incubated with $0.5 \times 10^{6} \mathrm{cpm}$ of radiolabeled $\mathrm{H} 1^{\circ} \mathrm{RNA}$. Competition experiments were performed in the presence of excess unlabeled H1 ${ }^{\circ}$ RNA (2:1), H3.3 RNA (2:1) or Cox IV RNA (5:1). At the end of the T1 RNase protection assay, RNA-protein covalent complexes were analyzed by SDS-PAGE and the gels were exposed to X-ray films for $16 \mathrm{~h}$. Representative films showing the RNA-protein complexes obtained in the absence (-) or in the presence of competing RNA are shown in the upper part of A and B. The lower parts of A and B are graphical representations of the statistical analysis of at least three independent experiments. Grey bars indicate mean values for each condition. SDs are also indicated (black bars).

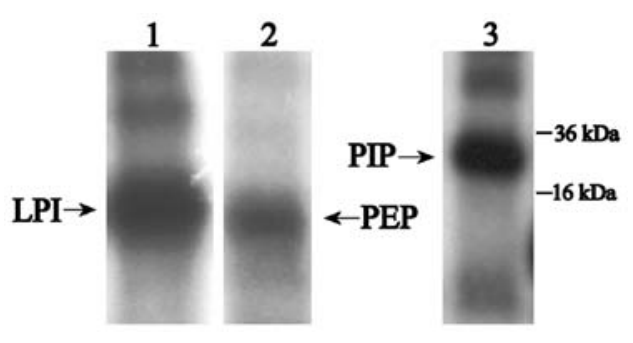

Figure 2. LPI and PEP-19 bind H3.3 RNA. LPI (lane 1), PEP-19 (lane 2) or PIPPin (lane 3, PIP) (50 ng each) was incubated with $0.5 \times 10^{6} \mathrm{cpm}$ radiolabeled H3.3 RNA. At the end of the T1 RNase protection assay, the RNA-protein covalent complexes were analyzed as described in Materials and methods and in Fig. 1 legend.

4:1 excess of the competing protein. Competition experiments aimed at analyzing interference effects of calmodulin on LPI and PEP19 binding to $\mathrm{H} 1^{\circ}$ RNA were performed in the presence of 1:1 or 4:1 excess of calmodulin. As a control of the interference experiments we used bovine serum albumin at the same concentration as calmodulin. 


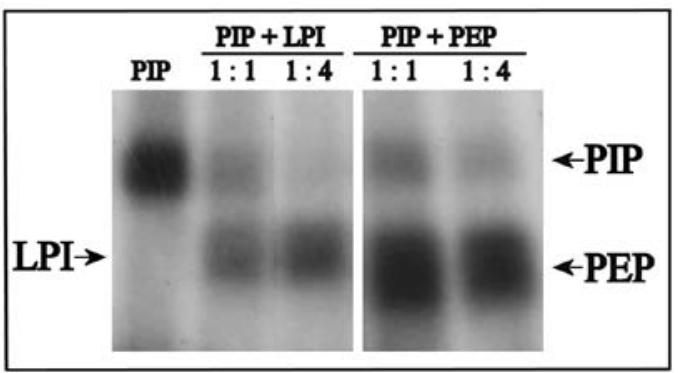

Figure 3. PEP-19 and LPI compete with PIPPin for $\mathrm{H} 1^{\circ}$ RNA binding. PIPPin (PIP) $(50 \mathrm{ng})$ were incubated with $0.5 \times 10^{6} \mathrm{cpm}$ of radiolabeled $\mathrm{H} 1^{\circ} \mathrm{RNA}$. Competition experiments were performed in the presence of a 1:1 or 1:4 excess of LPI or PEP-19 (PEP).

\section{Results}

RNA-binding activity of PEP-19 and LPI and specificity of $R N A$-binding. One possible way to identify RNA-binding proteins is to screen expression libraries with ${ }^{32} \mathrm{P}$-labeled RNA of interest. As reported elsewhere (15), we recently described a cDNA, isolated through such a screening and encoding a translational product that we called LPI, since it was found to be a rat-specific, alternative splicing variant of the already known PEP-19 peptide. The two proteins share the C-terminal half, which includes a calmodulin-binding domain. Recombinant LPI, as well as PEP-19 and PIPPin (CSD-C2), all from rat, were expressed in $E$. coli and purified from the insoluble fraction of the bacterial homogenate by chromatography. To confirm that LPI can bind $\mathrm{H} 1^{\circ}$ RNA, as suggested by the approach used to identify it, and to investigate PEP-19 binding properties, T1 RNase assays were performed, using in vitro transcribed
$\mathrm{H} 1^{\circ}$ RNA and $50 \mathrm{ng}$ of each protein. Both LPI (Fig. 1A) and PEP-19 (Fig. 1B) were able to bind rat $\mathrm{H} 1^{\circ}$ RNA, each forming a major complex which migrates with an apparent molecular weight of 14 and $12 \mathrm{kDa}$, respectively. In addition, to ascertain that the binding of LPI and PEP-19 to $\mathrm{H} 1^{\circ}$ RNA was specific, $\mathrm{T} 1 \mathrm{RNase}$ protection assays were performed in the presence of excess unlabeled RNAs. Unlabeled $\mathrm{H} 1^{\circ}$ RNA competed with his labeled counterpart in the binding reactions with both LPI (Fig. 1A) and PEP-19 (Fig. 1B). Unlabeled H3.3 RNA (Fig. 1) also competed with $\mathrm{H}^{\circ}$ RNA binding, suggesting an interaction of both proteins with both RNAs. In contrast, the 3'UTR of Cox IV RNA (Fig. 1) was unable to compete. Fig. 1 also shows a graphical representation of the statistical analysis of at least three independent experiments, for both proteins.

The ability of LPI and PEP-19 to bind H3.3 RNA was confirmed by direct T1 RNase protection assay (Fig. 2). Both LPI (lane 1) and PEP-19 (lane 2) strongly bound H3.3 RNA, suggesting an interaction of these proteins with both the $\mathrm{H} 1^{\circ}$ and H3.3 histone variants RNAs. Fig. 2 also shows H3.3 RNA binding to PIPPin, the RNA-binding activity of which has been previously reported $(12,13)$.

PEP-19 and LPI compete with PIPPin for H $1^{\circ}$ RNA-binding. Since both LPI and PEP-19 are able to bind the H1 ${ }^{\circ}$ RNA, we assessed their ability to compete with PIPPin for binding. Fig. 3 shows the results of these analyses: when used in equimolar amounts, LPI and PIPPin form roughly similar amounts of complexes. On the other hand, PEP-19 seems to bind $\mathrm{H} 1^{\circ}$ RNA more strongly than PIPPin.

Effect of calmodulin on PEP and LPI binding to $H 1^{\circ}$ RNA. Since both LPI and PEP are able to bind $\mathrm{H} 1^{\circ} \mathrm{RNA}$, we reasoned that the site involved in RNA binding might be in their common
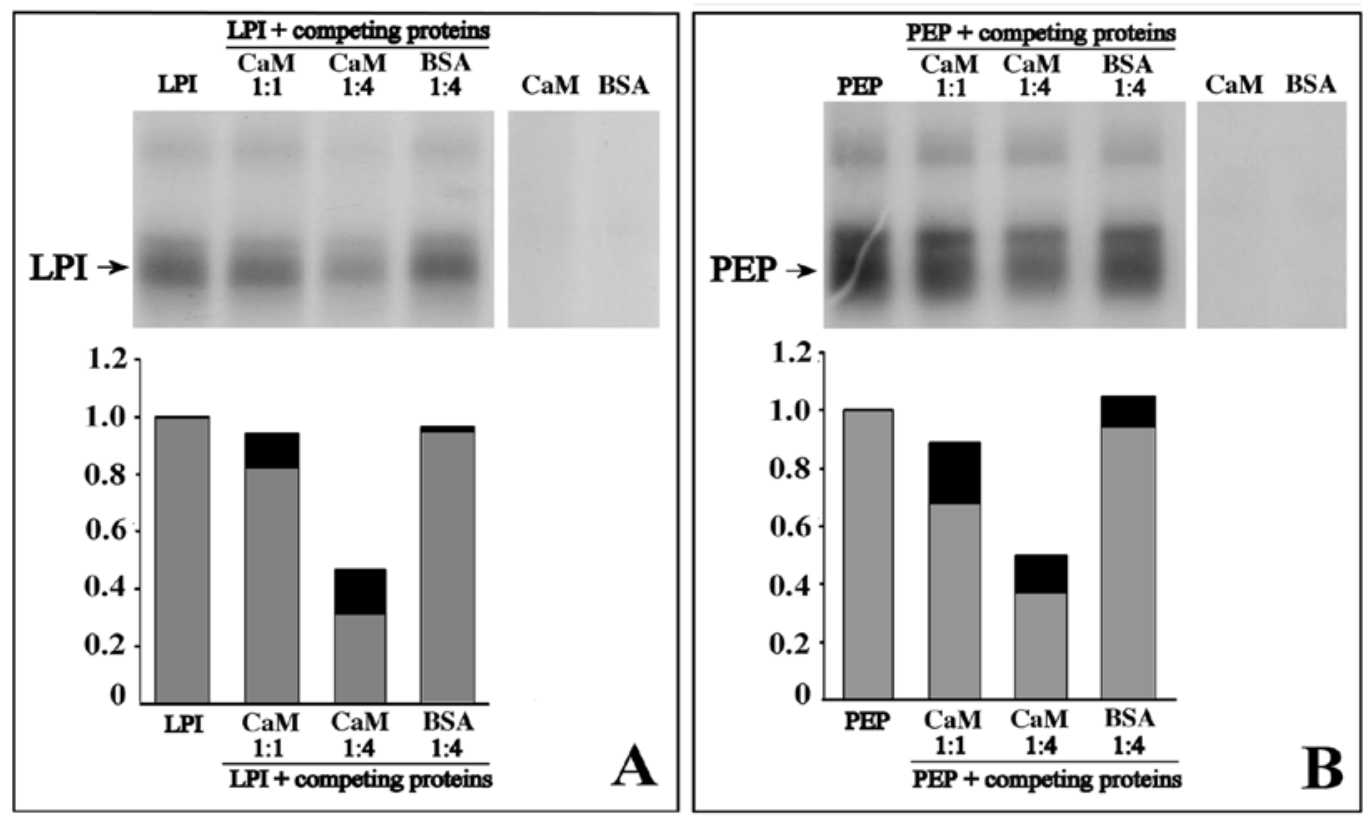

Figure 4. Calmodulin interferes with RNA binding to PEP-19 and LPI but does not bind RNA. (A) LPI or (B) PEP-19 (50 ng each) were incubated with radiolabeled $\mathrm{H} 1{ }^{\circ} \mathrm{RNA}$. Competition experiments were performed in the presence of $1: 1$ or $4: 1$ excess of calmodulin (CaM), or $4: 1$ excess of bovine serum albumin (BSA). At the end of the T1 RNase protection assay, RNA-protein covalent complexes were analyzed as described in the legend to Fig. 1. Representative films are shown in the upper part of A and B. The lower parts of A and B are graphical representations of the statistical analysis of at least three independent experiments. Grey bars indicate mean values for each condition. SDs are also indicated (black bars). 
C-terminal half. This part of both molecules also contains the calmodulin-binding site. We therefore, asked whether calmodulin can interfere with RNA binding. Calmodulin was able to reduce the binding of both proteins to RNA (Fig. 4). The interfering effect of calmodulin is specific, since the same amount of BSA did not compete. Graphical representation of the statistical analysis of at least three independent experiments are shown in Fig. 4, for both proteins.

The effect of calmodulin is not due to a putative ability to bind RNA: this protein, like BSA, was unable to form complexes with $\mathrm{H} 1^{\circ}$ RNA on its own (Fig. 4).

\section{Discussion}

Post-transcriptional regulation of RNA fate depends on the association with different sets of proteins (RNA-binding proteins, RBPs), which often contain different RNA-binding domains as well as additional domains involved in proteinprotein interactions (22). The RNA-RBP association can be modulated by environmental cues, both during development and in differentiated cells $(5,23)$.

During mammalian brain maturation, several classes of neurons accumulate both $\mathrm{H} 3.3$, a core histone $(9,10)$, and $\mathrm{H} 1^{\circ}$, a linker histone $(7,10)$. The concentration of the corresponding mRNAs decreases between embryonal Day 18 (E18) and postnatal Day 10 (P10), with an inverse correlation to the accumulation of the corresponding proteins. The observed differences are not due to modifications of gene transcription (24) and should depend on post-transcriptional regulation. The search of RBPs able to bind $\mathrm{H} 1^{\circ}$ - and/or H3.3-mRNA, and possibly involved in their metabolism, in the past led to the identification of three $\mathrm{H} 1^{\circ}$ RNA-binding proteins (p40, p70 and p110) (11) and to cloning of an $\mathrm{H} 3.3 / \mathrm{H} 1^{\circ}$ RNA-binding protein (PIPPin, also known as CSD-C2), which contain a cold-shock domain (13). More recently, a second protein has been cloned through an experimental approach based on screening of an expression cDNA library by a functional binding assay with a labeled, in vitro transcribed histone RNA (15). The novel cDNA corresponds to a splicing variant of the mRNA encoding PEP-19, an already known peptide which contains, in its C-terminal half, IQ motifs able to bind calmodulin. The new protein, that we called LPI, shares with PEP-19 the calmodulin-binding C-terminal half. The present study was therefore aimed, first of all, at investigating whether LPI and PEP-19 interact with $\mathrm{H} 1^{\circ} \mathrm{RNA}$. The analysis required preparation of recombinant PEP-19 and LPI, but, when produced in bacteria as 6 histidine-tagged, most of these proteins were in the inclusion bodies. It was therefore necessary to set an experimental procedure that, moving from the standard denaturation/refold protocols, could allow a high yield of functional protein. In particular, according to standard protocols, proteins are finally dialyzed. During this step, however, most protein aggregated and precipitated. We found that replacement of the final dialysis with a filtration through an equal volume of G-100 Sephadex could restore solubility of the proteins. Moreover, solubility is maintained even after repeated freeze-thaw cycles.

LPI and PEP-19 prepared through this procedure, like the previously identified PIPPin protein, are able to bind both $\mathrm{H} 1^{\circ}$ and H3.3 RNAs. The fact that all three proteins, which are also able to compete for binding to RNA, recognize both histone variant RNAs suggest the existence of a common regulation of the synthesis of replacement histones. Once we had demonstrated that both LPI and PEP-19 are able to bind $\mathrm{H} 1^{\circ}$ and H3.3 RNAs, we assessed the possible interfering effect of calmodulin on RNA-binding. The finding that calmodulin actually reduces binding of RNA to both proteins sheds light on the putative signals which could regulate expression of histone variants in the brain. Calcium-dependent signals are indeed of particular importance in the nervous system, and deregulated increases in intracellular ionized calcium can result in neuronal damage and death. Many of the effects of calcium are mediated by calmodulin, and the brain contains tissue-specific peptides (such as PEP-19, neurogranin and neuromodulin) which seem to act as calmodulin antagonists (17). Moreover, it has been recently suggested that PEP-19 is a critical determinant of synaptic plasticity (25). Our discovery that PEP-19 and LPI bind $\mathrm{H} 1^{\circ}$ histone mRNA and that this binding is affected by calmodulin suggests that, in the brain, post-transcriptional regulation of $\mathrm{H} 1^{\circ}$ histone synthesis may be regulated by calcium signals, and perhaps by neuronal activity.

\section{Acknowledgements}

This study was supported by the Italian Ministero dell'Istruzione e della Ricerca (MIUR) and by the University of Palermo (Università degli Studi di Palermo, ex $60 \%$ to ID), Italy. P.S. received a PhD studentship from the University of Palermo.

\section{References}

1. Kornberg RD and Lorch Y: Twenty-five years of the nucleosome, fundamental particle of the eukaryote chromosome. Cell 98: 285-294, 1999.

2. Ahmad K and Henikoff S: The histone variant H3.3 marks active chromatin by replication-independent nucleosome assembly. Mol Cell 9: 1191-1200, 2002.

3. Gao FB: Messenger RNAs in dendrites: localization, stability, and implications for neuronal function. Bioessays 20: 70-78, 1998.

4. Kuhl D and Skehel P: Dendritic localization of mRNAs. Curr Opin Neurobiol 8: 600-606, 1998.

5. Derrigo M, Cestelli A, Savettieri G and Di Liegro I: RNA-protein interactions in the control of stability and localization of messenger RNA. Int J Mol Med 5: 111-123, 2000.

6. Gjerset R, Gorka C, Hasthorpe S, Lawrence JJ and Eisen H: Developmental and hormonal regulation of protein $\mathrm{H} 1^{\circ}$ in rodents. Proc Natl Acad Sci USA 79: 2333-2337, 1982.

7. Cestelli A, Castiglia D, Di Liegro CM and Di Liegro I: Qualitative differences in nuclear proteins correlate with neuronal terminal differentiation. Cell Mol Neurobiol 12: 33-43, 1992.

8. Ponte I, Martinez P, Ramirez A, Jorcano JL, Monzó M and Suau P: Transcriptional activation of histone $\mathrm{H} 1$ zero during neuronal terminal differentiation. Brain Res Dev Brain Res 80: 35-44, 1994.

9. Pina B and Suau P: Changes in histones H2A and H3 variant composition in differentiating and mature rat brain cortical neurons. Dev Biol 123: 51-58, 1987.

10. Castiglia D, Cestelli A, Scaturro M, Nastasi T and Di Liegro I: $\mathrm{H} 1^{\circ}$ and $\mathrm{H} 3.3 \mathrm{~b}$ mRNA levels in developing rat brain. Neurochem Res 19: 1531-1537, 1994.

11. Scaturro M, Nastasi T, Raimondi L, Bellafiore M, Cestelli A and Di Liegro I: $\mathrm{H} 1^{\circ} \mathrm{RNA}-$ binding proteins specifically expressed in the rat brain. J Biol Chem 273: 22788-22791, 1998.

12. Castiglia D, Scaturro M, Nastasi T, Cestelli A and Di Liegro I: PIPPin, a putative RNA-binding protein specifically expressed in the rat brain. Biochem Biophys Res Commun 218: 390-394, 1996.

13. Nastasi T, Scaturro M, Bellafiore M, Raimondi L, Beccari S, Cestelli A and Di Liegro I: PIPPin is a brain-specific protein that contains a cold-shock domain and binds specifically to $\mathrm{H} 1^{\circ}$ and H3.3 mRNAs. J Biol Chem 274: 24087-24093, 1999. 
14. Nastasi T, Muzi P, Beccari S, Bellafiore M, Dolo V, Bologna M, Cestelli A and Di Liegro I: Specific neurons of brain cortex and cerebellum are PIPPin positive. Neuroreport 11: 2233-2236, 2000.

15. Sala A, Scaturro M, Proia P, Schiera G, Balistreri E, AflaloRattenbach R, Créau N and Di Liegro I: Cloning of a rat-specific long PCP4/PEP19 isoform. Int J Mol Med 19: 501-509, 2007.

16. Ziai R, Pan YC, Hulmes JD, Sangameswaran L and Morgan JI: Isolation, sequence, and developmental profile of a brain-specific polypeptide, PEP-19. Proc Natl Acad Sci USA 83: 8420-8423, 1986.

17. Slemmon JR, Morgan JI, Fullerton SM, Danho W, Hilbush BS and Wengenack TM: Camstatins are peptide antagonists of calmodulin based upon a conserved structural motif in PEP-19, neurogranin, and neuromodulin. J Biol Chem 271: 15911-15917, 1996.

18. Johanson RA, Sarau HM, Foley JJ and Slemmon JR: Calmodulinbinding peptide PEP-19 modulates activation of calmodulin kinase II in situ. J Neurosci 20: 2860-2866, 2000.

19. Markossian KA and Kurganov BI: Protein folding, misfolding, and aggregation. Formation of inclusion bodies and aggresomes. Biochemistry 69: 971-984, 2004.
20. Raimondi L, D'Asaro M, Proia P, Nastasi T and Di Liegro I: RNA-binding ability of PIPPin requires the entire protein. J Cell Mol Med 7: 35-42, 2003

21. Castiglia D, Gristina R, Scaturro M and Di Liegro I: Cloning and analysis of cDNA for rat histone $\mathrm{H} 1^{\circ}$. Nucleic Acids Res 21: 1674, 1993.

22. Burd CG and Dreyfuss G: Conserved structures and diversity of functions of RNA-binding proteins. Science 265: 615-621, 1994.

23. Hall KB: RNA-protein interactions. Curr Opin Struct Biol 12: 283-288, 2002.

24. Scaturro M, Cestelli A, Castiglia D, Nastasi T and Di Liegro I: Posttranscriptional regulation of $\mathrm{H} 1$ zero and $\mathrm{H} 3.3 \mathrm{~B}$ histone genes in differentiating rat cortical neurons. Neurochem Res 20: 969-976, 1995.

25. Wei P, Blundon JA, Rong Y, Zakharenko SS and Morgan JI: Impaired locomotor learning and altered cerebellar synaptic plasticity in pep-19/pcp4-null mice. Mol Cell Biol 31: 2838$2844,2011$. 